\title{
Signal analysis of a polarimetric fiber optic sensor for dynamic strain monitoring
}

\author{
Marcin S. Bieda*, Andrzej W. Domański, Dawid Kuchta, Piotr Lesiak, and Tomasz R. Woliński \\ Faculty of Physics, Warsaw University of Technology, Koszykowa 75, 00-662 Warszawa \\ Received May 09, 2014; accepted July 01, 2014; published July 01, 2014
}

\begin{abstract}
Polarimetric fiber optic sensors have many advantages over other fiber optic sensors and strain gauges. As opposed to Fiber Bragg Grating sensors, polarimetric fiber sensors can be constructed with a very simple, portable and robust experimental setup. In the paper we propose two methods of analyzing a polarimetric sensor output signal. The first method is based on depolarization phenomena, and calculates the degree of polarization (DOP), the second one uses Fast Fourier Transform to analyze dynamic sensor response. The usefulness of both methods has been shown and experimentally proven.
\end{abstract}

Optical fiber sensors are often used to measure dynamic strain in various materials. They offer significant advantages over electric and electronic strain gauges, especially when used in composite materials. Optical fibers can be easily embedded into such materials, thus fibers can be easily used to monitor health, internal strain and temperature of the structure during their operation. Fiber Bragg grating (FBG) sensors are the most popular strain sensors. However, polarimetric sensors offer many advantages compared to FBGs. They offer a simpler costeffective setup as well as a possibility to measure distributed strain, which could be easily omitted by FBG sensor [1-3]. In many applications, dynamic forces are much more important than static pressure.

Fiber optic polarimetric sensors measure the change in the state of polarization (SOP) of output light of a sensor caused by birefringence changes of the fiber. In order to measure strain, a highly birefringent (HB) optical fiber is embedded into a composite material. If longitudal strain $\varepsilon$ is applied to the sensing part of the fiber, beat length parameter $L_{B}$ is modified according to the formula [4]:

$$
L_{B}(\varepsilon)=\frac{L_{B 0}}{1+\frac{\varepsilon \cdot L_{B 0}}{T_{\varepsilon} L}},
$$

where $T_{\varepsilon}$ represents the sensitivity of a sensor, and is defined as the strain value which produces a phase shift equal to $2 \pi . L_{B O}$ is the beat length of the fiber without applied strain. Due to the birefringence of the fiber, the degree of polarization (DOP) is reduced depending on the coherence of a light source. In order to maintain reasonably high DOP and reduce the influence of temperature changes, compensation fiber is added with its axes crossed at $90^{\circ}$ to the sensing part. The light from the source is linearly polarized at $45^{\circ}$ to the fast axis of the fiber [5], so that both polarization modes are evenly exited, and output intensity is defined as in equation [6]:

$$
I_{\text {out }}=\text { const } \cdot\left[1+\exp \left[\left(-\frac{\lambda \varepsilon}{L_{c} T_{\varepsilon}}\right)^{\alpha}\right] \cos \left(\frac{2 \pi \varepsilon}{T_{\varepsilon}}\right)\right],
$$

where $L_{C}$ is the temporal coherence length of a light source, $\alpha=1$ for Lorenzian spectrum and $\alpha=2$ for Gaussian spectrum. According to Eq. (2), the output signal from a compensated polarimetric sensor is sinusoidal. The amplitude of the sin-like function is equal to the dynamic range of an output signal, which is determined by an exponential component. Signal amplitude corresponds to light depolarization due to the applied strain, which highly depends on the spectrum width of a light source [7]. The experimental setup is shown in Fig. 1.

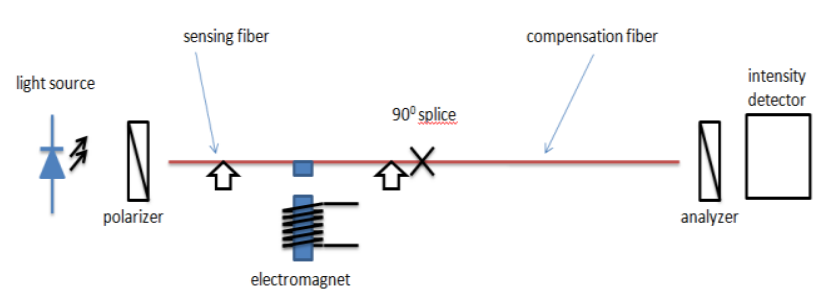

Fig. 1. Compensated polarimetric fiber optic strain sensor.

The sensor architecture presented in Fig. 1 is very simple and cost-effective, yet provides an accurate output signal measurement. Furthermore, the light source and detector are small and portable, compared to a polarimeter or an optical spectrum analyzer, so it is possible to measure strain in composite materials in-situ. The use of an electromagnet allowed us to characterize the response of a fiber optic, polarimetric sensor to precisely control dynamic strain. 


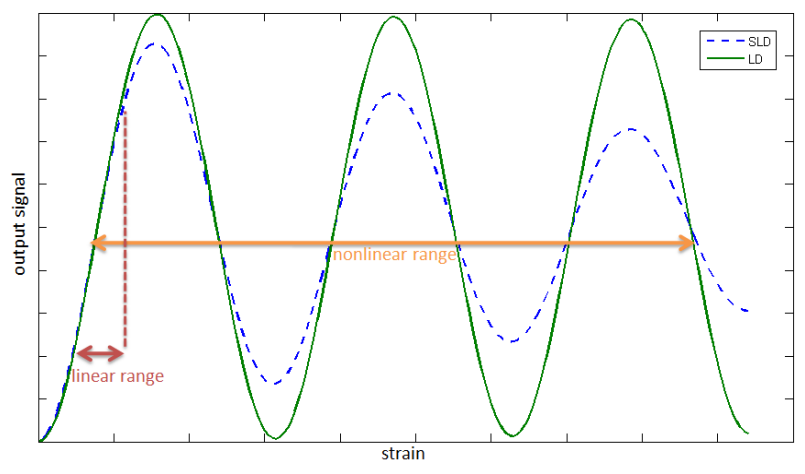

Fig. 2. Polarimetric sensor response for laser diode (LD), and superluminescent diode (SLD)

The proposed polarimetric sensor can operate in two different ranges of the signal presented in Fig. 2. If maximal applied strain is small in relation to the fiber sensitivity $T \varepsilon$, then the output signal is quasi linear. The output signal from the sensor is easy to analyze, but has a small dynamic range. Furthermore, the operating range of the sensor must be carefully chosen in the middle of the slope of a sin-like function. As a result it is often more desirable to operate in a nonlinear range of the sensor. The output signal has a greater dynamic range, but it requires post-processing in order to calculate strain.

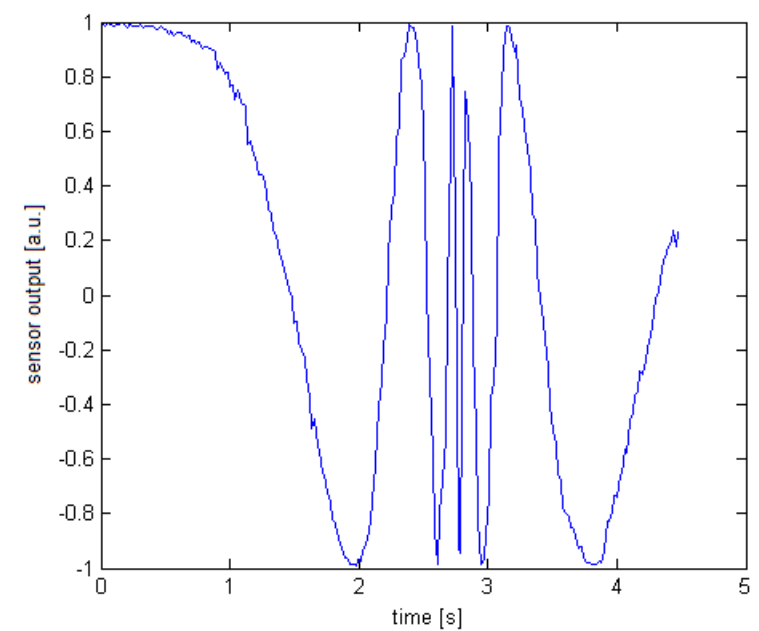

Fig. 3a. Polarimetric sensor response for triangular shape strain pulse: non linear,

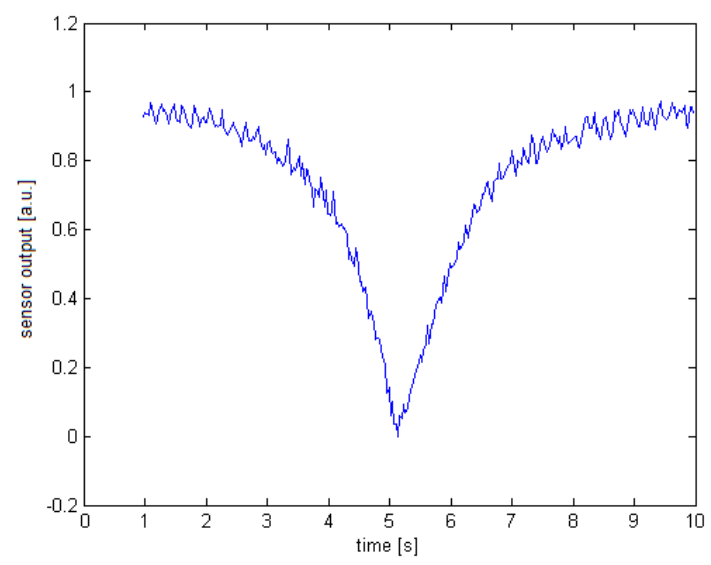

Fig. 3b. Polarimetric sensor response for triangular shape strain pulse: linear

\section{A. Depolarization analysis}

In many applications it is not possible to use a polarimetric sensor in a linear working range of operation. When analyzing a nonlinear, periodic signal, additional information is needed in order to calculate strain. Various methods have been proposed to solve this problem, for example a two wavelength quadrature signal readout [8], which requires at least two light detectors. With a single detector it is still possible to measure strain by taking advantage of depolarization phenomena. By measuring the amplitude of sensor output, the DOP can be calculated, and used as a coarse strain indicator. The phase of the periodic signal could then be used to determine strain more precise.

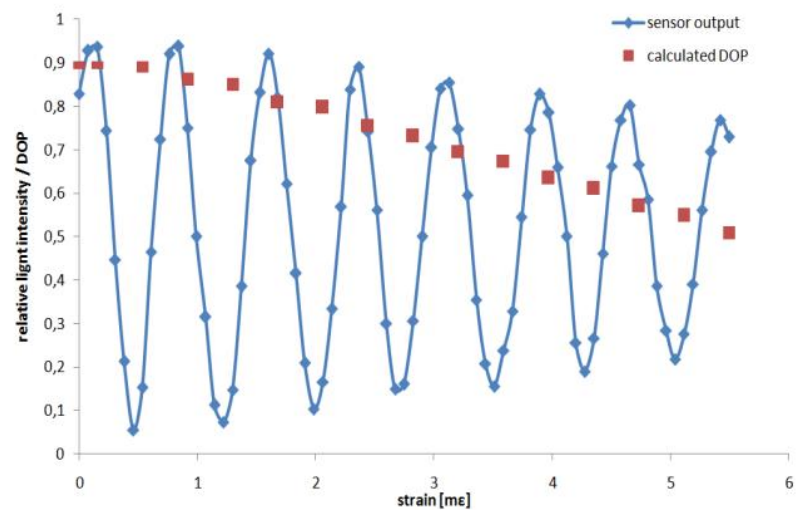

Fig. 4. Output signal from polarimetric strain sensor with calculated DOP 
Experimental results are presented in Fig. 4. Superluminescent diode $(\lambda=1295 \mathrm{~nm}, \Delta \lambda=50 \mathrm{~nm})$ were used as a light source, a $350 \mathrm{~mm}$ length HB bow-tie fiber was used in both sensing and compensation part of sensor.

\section{B. Fast Fourier Transform-based signal analysis}

Another approach to calculate dynamic strain is based on the use of a Fast Fourier Transform (FFT). In the setup shown in Fig. 1 a triangular signal was applied to the electromagnet. A sample plot of light intensity versus time is shown in Fig. 3a. The signal was analyzed with a windowed FFT, in order to calculate spectral response. The fundamental frequency is related to linear sensor response, amplitudes of higher harmonics depend on nonlinear sensor signal. Fig. 5 presents the results of an FFT integrated over the higher harmonics in relation to the applied strain. It is visible that there is a linear relationship between them, but the proposed method needs to be improved in terms of accuracy.

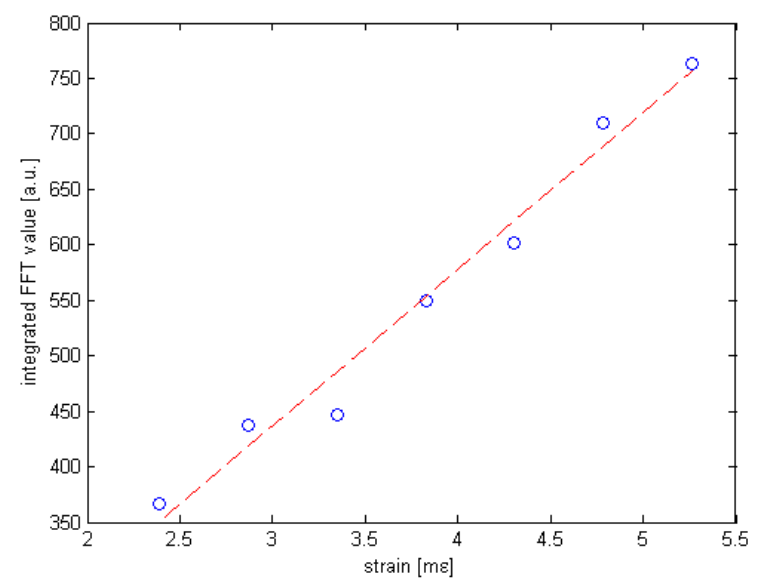

Fig. 5. Integrated FFT versus amplitude of applied dynamic strain.

In conclusions, both of the presented methods of the analysis are valuable in demodulating the output signal coming out of the polarimetric fiber strain sensor. Depolarization of light can be used to determine strain in a coarse way, a fine strain value could be read from the phase of a sin-like signal. In this method, the length of the optical fiber and coherence of a light source must be carefully chosen, in order to measure depolarization phenomena. The second method is based on integrating FFT peak values. The experiment has shown that by using this method, linear dependency with respect to the applied strain pulse can be obtained. Both methods could be applied to monitor dynamic strain in various materials. Future efforts have to be taken in order to combine the results from the two presented methods and improve their accuracy.
Research leading to the paper was supported by the National Centre for Research and Development (NCBiR) under the grant PBS1/B5/20/2012.

\section{References}

[1] K. Schroeder, W. Ecke, J. Apitz, E. Lembke, G. Lenschow, Meas. Sci. Technol. 17, 1167 (2006).

[2] G. Hegde, A. Asundi, NDT\&E International 39, 320 (2006).

[3] G. Wehrle, P. Nohama, H. J. Kalinowski, P. I. Torres, L.C.G.Valente, Meas. Sci. Technol. 12, 805 (2001).

[4] W.J. Bock, A.W. Domanski, T.R. Wolinski, Appl. Optics 29, 3484 (1990).

[5] A.W. Domański, M. Bieda, P. Lesiak, P. Makowski, M. Szeląg, T. Poczęsny, K. Prokopczuk, P. Sobotka, M. Chychłowski, M. Sierakowski, T.R. Woliński, Acta Phys. Polonica A. 124, 399 (2013).

[6] P. Lesiak, G. Rajan, Y. Semenova, G. Farrell, A. Boczkowska, A.W. Domański, T.R. Woliński, Phot. Lett. Poland 3, 140 (2010).

[7] R.A. Chipman, Proc. SPIE 5158, 184 (2003).

[8] N. Furstenau, M. Schmidt, W. J. Bock, W. Urbanczyk, App. Opt. 37, 663 (1998). 